\title{
Zeit für ein neues Normalarbeitsverhältnis!
}

\author{
ULRICH MÜCKENBERGER
}

$D$ ie Krise des mittlerweile erodierten Normalarbeitsverhältnisses hat viel mit der Arbeitszeitfrage zu tun. Solange feste und klar abgegrenzte Arbeitstage, freie Wochenenden und jährliche Urlaubs- und Weihnachtspausen das kollektive Zusammenleben strukturierten, war Solidarität eine Frucht gemeinsamer Arbeits- und Nichtarbeitserfahrung. Arbeit hat sich aber zerfasert, abgrenzbare Konturen verloren, ist zerstückelt, flexibel zusammengesetzt, intensiviert, „desynchronisiert" worden. Die gelebte Normalität ist zerbröselt und hat Individualisierung hinterlassen. Der Soziologe Ulrich Beck hat Risiken und Chancen der Individualisierung ausfindig gemacht: Risiken der Vereinzelung und des Verlusts sozialer Netze, Chancen neuer Alltags- und Lebensentwürfe, neuer Natur-, Geschlechter- und Generationenbeziehungen.

Heute über ein neues Normalarbeitsverhältnis zu sprechen, heißt, nicht in standardisierte und patriarchalische Regelungsformen, die vormals die Normalität ausmachten, zurückzufallen. Wir sollten die Chancen des gewandelten Verständnisses von Arbeit zu verstärken versuchen. Dieses Verständnis geht von Individuen nicht als schutzlosen Opfern, sondern als mit Bürgerrechten ausgestatteten Gestaltern aus; von Menschen, die neben Erwerbsarbeit in vielerlei gesellschaftliche und familiäre Aufgaben und Engagements eingebunden sind; von Menschen, die unterschiedlich sind und Wert auf ihre „Diversität“"legen; von Menschen, die nicht Freiheit gegen Schutz eintauschen wollen, sondern Autonomie und Verantwortlichkeit für ihre Lebensführung verlangen und dabei Verlässlichkeit und Schutz beanspruchen.

Welche Bedingungen müssen im Arbeits- und außererwerblichen gesellschaftlichen Leben gegeben sein - also geschaffen werden -, um Chancen neuer Selbst- und Gesellschaftsfindung verwirklichen zu helfen? Wie können wir zum Lernen und Gelingen neuer Lebensentwürfe beitragen und dafür sorgen, dass diese nicht zulasten anderer Menschen und folgender Generationen oder der Natur gehen? Die gesuchte neue Normalität stellt also keine „Gegebenheit", sondern eine Perspektive dar - den zukunftsbezogenen Orientierungspunkt für gestaltendes Handeln in der Gegenwart: für Regierungen und Parlamente, Tarifvertragsparteien, Wissenschaftlerinnen und Wissenschaftler, betriebliche Sozialpartner, einzelne Mitarbeiter und Vorgesetzte, die heute entscheiden und so das Leben von morgen gestalten.

Wenn wir so verstandener Normalität - ihren Bedingungen und Gestaltungserfordernissen - nachgehen, sie zu fassen und zu konkretisieren versuchen, sind wir unweigerlich mit Fragen der Zeit, der Zeitverfügung und der Zeitorganisation konfrontiert. Wo standardisierte und typisierte Alltagsverläufe durch individualisierte und flexibilisierte ersetzt werden, sind Zeiten für Kommunikation und Koordination schon für die alltägliche Arbeitsverrichtung unverzichtbar. Das gilt besonders bei Dienstleistungen, die in „Ko-Produktion“ zwischen Beschäftigten und Dienstleistungsnutzern erbracht und konsumiert werden. Zeit ist Grundbedingung von Lern- und Entwicklungsprozessen innerhalb wie außerhalb der be- zahlten Arbeit. Die Frage der Zeitverfügung ist aufgeworfen, wenn wir Beschäftigte auch im außerbetrieblichen Bereich als Gestaltende anerkennen und unterstützen wollen. Der Betrieb muss da seinen Monopolanspruch auf Verfügung über die Lebenszeit erwachsener Menschen aufgeben: Er muss das kostbare Gut Zeit teilen mit anderen Lebenssphären - wie Familie, Beziehung, Ehrenamt, Nachbarschaft, Politik, Kultur -, die zu ihrem Gelingen Zeit verlangen. Dabei fehlen darf nicht die Einwilligung derer, um deren Lebenszeit es sich handelt - was wieder Zeit für Partizipation erfordert. Man sieht: In der individualisierten Gesellschaft tun sich neben nicht gekannten Zeitbedarfen Zeitkonflikte auf, deren fruchtbare Schlichtung wiederum Zeit erfordert.

Die Brisanz der zeitpolitischen Folgerungen aus diesen Überlegungen wird atemberaubend, wenn wir sie mit der Zeitkultur konfrontieren, die in den meisten Betrieben, Verwaltungen, bei Dienstleistungserbringern wie Krankenhäusern, Schulen usw. vorherrscht. Meist wird mit Zeit unachtsam umgegangen - Tendenz dramatisch zunehmend. Zeit wird als knappe Ressource gnadenlos rationiert und durchrationalisiert. Deshalb kumulieren Zeitkonflikte gerade da, wo dem betriebswirtschaftlichen ein Zeitverständnis begegnet, das menschlichen Eigenzeiten Raum gibt.

Eine Normalisierung der Arbeitsverhältnisse erfordert heute zumindest zwei radikale zeitpolitische Einschnitte. Dem zunehmenden Raubbau an der Lebenszeit von Menschen muss durch entschleunigende Gegenwehr und zeitachtsame Ausgestaltung der Arbeitsbedingungen wirksamer als je zuvor entgegengetreten werden. Das erfordert die Verankerung von Reflexionszeiten („Zeit für Zeit“) und mehr direkter individueller Beteiligung im Arbeitsalltag. Und: In der Arbeitszeitgestaltung müssen viel stärker die zeitlichen Bedürfnisse der Menschen als Bürgerinnen und Bürger innerhalb wie außerhalb des Erwerbslebens wirksam gemacht werden. Wann gehört zur Normalität von Arbeitsverhältnissen, dass im Erwerbsalter relevante Zeiteinheiten für andere gesellschaftliche Notwendigkeiten (vor allem Care-Tätigkeit, aber auch Bildung, Muße etc.) zur Verfügung stehen, die mit differenziertem Entgeltersatz verbunden sind und je nach Lebenslage "gezogen" werden können? Klar: Diese Normalisierung ist einschneidend - aber erst sie kann Arbeit humanisieren und die Kluft zwischen Arbeit und Leben überwinden helfen.

ULRICH MÜCKENBERGER, Professor em. für Rechts- und Politikwissenschaft der Universität Hamburg, hat derzeit eine Forschungsprofessor am Zentrum für Europäische Rechtspolitik der Universität Bremen und ist Vorsitzender der Deutschen Gesellschaft für Zeitpolitik. Arbeitsschwerpunkte: Deutsches und internationales Arbeitsrecht, Globalisierung und Governance sowie Zeitforschung und -politik.

mueckenb@uni-bremen.de 